\title{
Mitochondrial transplantation in humans: "magical" cure or cause for concern?
}

\author{
Edoardo Bertero, ${ }^{1}$ Christoph Maack, ${ }^{1}$ and Brian O'Rourke ${ }^{2}$ \\ 'Comprehensive Heart Failure Center, University Clinic Würzburg, Germany. 'Division of Cardiology, Department of Medicine, Johns Hopkins University, Baltimore, Maryland, USA.
}

M itochondrial transplantation is a therapeutic approach developed by McCully and colleagues that entails the injection of healthy mitochondria harvested from unaffected tissue into an ischemic organ of the same subject (1). It has recently been applied to human pediatric patients with myocardial ischemia (2), receiving widespread media attention accompanied by sensationalistic claims on its mechanism of action, e.g.: (a) after injection into the heart, "mitochondria moved like magnets to the proper places in the cells and began supplying energy;" and (b) after infusion into the coronary arteries, "somehow the organelles will gravitate almost magically to the injured cells that need them and take up residence" (3).

According to the purported mechanism of action, the mitochondria must, seemingly, perform three "magic tricks." First, the mitochondria must survive transfer from an intracellular environment to an extracellular one with high $\mathrm{Ca}^{2+}$ concentrations. Second, if they survive, mitochondria must produce ATP that is able to enter cardiac myocytes to support contraction. Third, enough mitochondria must pass through the cell membrane to contribute to ATP production by the host cell. A corollary to trick number three is a variation in which the mitochondria are injected into the bloodstream and somehow pass through the endothelial vascular permeability barrier, migrate into the interstitium, and are incorporated into the dysfunctional target tissue. Given the rapid translation of this method to the clinic, it behooves us to determine whether these extraordinary claims are convincingly supported.

\section{Preclinical evidence of protection from myocardial ischemia-reperfusion}

McCully et al. first validated mitochondrial transplantation in animal models of ischemia-reperfusion (4-6). Isolated mitochondria from autologous skeletal muscle or human cardiac fibroblasts were injected epicardially into the ischemic heart one minute before reperfusion (4, 5 ) or delivered by vascular perfusion through the coronary arteries immediately after reperfusion (6). The authors noted decreased ROS formation, necrotic and apoptotic cell death, and smaller infarcts in hearts with injection of mitochondria (4-6). Within 10 minutes, they noted improvements in regional hypokinesis, left ventricular hemodynamics, and, within hours, higher ATP content with benefits maintained up to 21 to 28 days after reperfusion (4-6). Injection of nonviable mitochondria, mitochondrial components, or administration of exogenous ATP or ADP in the ischemic area had no effect (4). They attributed the benefits to an increase in ATP production from the transplanted mitochondria and, within a longer time frame, to the upregulation of enzymes involved in mitochondrial energy metabolism (5). In a study in pigs, a similar protocol reduced infarct size from $13 \%$ to $5 \%$ of the area at risk (7), but, interestingly, the protection was not dependent on the dose $-200,000$ mitochondria per gram of tissue were as effective as 20 million (7).

In our view, many important questions have not been adequately addressed in the rush to rapidly translate this procedure to clinical application (Figure 1).
Conflict of interest: CM declares the following conflicts that are unrelated to the article: honoraria for scientific advice and speaking fees from Servier (tier 1) and honoraria for speaking fees from Bayer, Boehringer Ingelheim, Berlin Chemie, Bristol Myers Squibb, Daiichi Sankyo, Novartis, and Pfizer (tier 2).

Reference information: / Clin Invest. 2018;128(12):5191-5194. https://doi.org/10.1172/JCI124944.
How do mitochondria survive high extracellular $\mathrm{Ca}^{2+}$ concentrations?

Mitochondria are highly permeable to $\mathrm{Ca}^{2+}$ (8), and when placed in micromolar concentrations of $\mathrm{Ca}^{2+}$, mitochondrial $\mathrm{Ca}^{2+}$ overload induces permeability transition pore formation in the inner membrane. Pore opening allows large molecules to leak out of the matrix, causing massive osmotic mitochondrial swelling, rupture of the outer membrane, and release of proapoptotic factors into the cytosol. In the above-mentioned studies, mitochondria were isolated and tested for function in a standard $\mathrm{Ca}^{2+}$ free respiration buffer (9). Subsequently, the mitochondrial suspension was injected directly into the myocardium. Prelabeled mitochondria were found embedded along the needle track of the injection, in close proximity to cardiac myocytes, but not inside them (4). More recently, mitochondria were resuspended in physiological buffer and then delivered via the coronary arteries during reperfusion (6). In these scenarios, mitochondria are exposed to high $\mathrm{Ca}^{2+}$ concentrations $(\sim 1.8 \mathrm{mM})$ in the blood or the extracellular fluid in the interstitium. It is extremely unlikely that the mitochondria could withstand these conditions without undergoing a permeability transition. Indeed, there is no direct evidence that the injected mitochondria remain viable after injection. Likewise, mitochondria prelabeled with an iron compound and injected into porcine hearts (7) were detected by MRI up to four weeks after injection; however, this method does not confirm the viability of the mitochondria. For example, phagocytosis of cellular debris by macrophages results in sustained iron signals for weeks after cardiac injection of labeled stem cells, even though the stem cells are no longer alive (10). A simple test to rule out the possibility that mitochondria are destroyed under the conditions of injection would be to measure mitochondrial respiration of the donor 


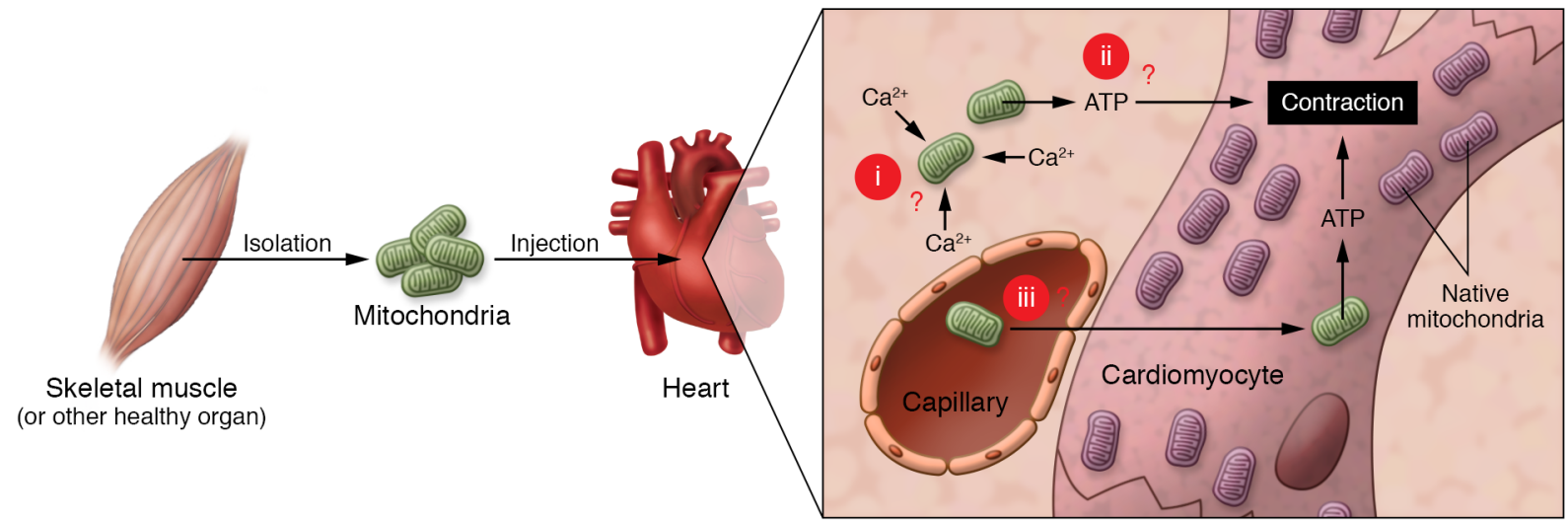

Figure 1. The proposed mechanism of action of mitochondrial transplantation in myocardial ischemia requires that the mitochondria perform three "magic tricks," which raises several critical questions. (i) For trick number one, the mitochondria must survive transfer from an intracellular environment to an extracellular one. This raises the question of how mitochondria survive high extracellular $\mathrm{Ca}^{2+}$ concentrations. (ii) For trick number two, mitochondria must produce ATP to support contraction. We ask: how can extracellular mitochondria produce ATP and how can extracellular ATP support contraction? (iii) For trick number three, a sufficient number of mitochondria must pass through the cell membrane to contribute to ATP production by the host cell. This prompts the questions of whether and how viable mitochondria actually enter myocytes and cross endothelial/vascular barriers, and do enough mitochondria do so to make a difference for ATP production?

mitochondria after resuspending them in high $\mathrm{Ca}^{2+}$ physiological buffer, which the McCully group has not done.

\section{How can extracellular mitochondria supply ATP for myofilament contraction?}

Let's consider for a moment that the mitochondria can survive in high $\mathrm{Ca}^{2+}$ conditions, avoid immune cell defenses, cross endothelial barriers, and end up in close proximity to the cardiac myocytes. In the heart, the total ATP pool is turned over in less than one minute, requiring enormous and well-tuned ATP production by thousands of mitochondria within each cardiac myocyte (11). Mitochondrial transplantation at the time of reperfusion of rabbit hearts improved cardiac function within minutes, while integration of mitochondria into cardiomyocytes took hours (5). The authors concluded that the injected mitochondria provided needed ATP to support cardiac contraction during early reperfusion. Notably, before mitochondria produce ATP, glucose and fatty acids must be converted to pyruvate and fatty acyl-coenzyme A (CoA), respectively, in the cytoplasm. It is unclear how, after injection into the extracellular space, mitochondria could convert primary substrates to the common Krebs cycle intermediate in the matrix, acetyl CoA, to generate ATP in the absence of the cytosol or how the myofilaments could access ATP generated outside the cell.

\section{How do mitochondria enter cardiac myocytes?}

McCully and colleagues showed evidence that isolated mitochondria could be taken up by cells in culture over an 8- to 12-hour time period and were not associated with endosomal or autophagic structures (5). The mitochondria also had a functional effect on the cells, as determined by the increased oxygen consumption and ATP measurements $(5,12)$. In human induced pluripotent stem cell-derived cardiac myocytes and human cardiac fibroblasts coincubated with labeled mitochondria (13), isolated mitochondria survived and were incorporated into cultured cells, escaped lysosomal degradation, and fused with the endogenous mitochondrial network (13). Thus, it could be argued that isolated mitochondria can survive in an extracellular culture medium and be taken up by cells in vitro. This process, called "mitochondrial transformation" was described previously in other laboratories (14), and it typically involves preparing mitochondria in media containing excess $\mathrm{Ca}^{2+}$ buffer and adding an aliquot to the culture dish. The final $\mathrm{Ca}^{2+}$ concentration in the dish is not measured, hence, it is unknown whether the conditions for mitochondrial transformation in cell culture prevent permeability transition pore activation. Internalization of functional mitochondria was demonstrated (15, 16), most convincingly by the restoration of mitochondrial respiration in rhoO cells, in which the native mitochondria were rendered nonfunctional by depletion of mitochondrial DNA (17). Although these data support the idea that mitochondrial transformation is possible in cultured cells, it is still unproven whether mitochondria remain viable and incorporate into myocytes of the heart under the conditions used by McCully and colleagues.

\section{How do the few mitochondria that enter cardiac myocytes produce sufficient ATP for contraction?}

According to the reports, $3 \%-7 \%$ of injected mitochondria are internalized by cardiac myocytes $(5,12,13)$. Even if the few mitochondria that colocalized with myocytes were inside them and capable of respiration, it is still hard to understand how this small number would make a difference in ATP production, given that there are several thousand mitochondria per myocyte. Moreover, in the pig study, immunostaining of a representative tissue section showed only three human mitochondria in an entire field of myocytes (7). In perfused rabbit hearts (6), high efficiency for mitochondrial transplantation was also claimed after coronary perfusion with human mitochondria. Despite widespread distribution of mitochondria visualized by PET imaging, histological tissue sections revealed an extremely sparse distribution of human mitochondria, mainly associated with the vasculature and 
interstitial spaces near capillaries, with the rare appearance of a mitochondrion close to a cardiac myocyte. It is quantitatively untenable to suggest that so few integrated mitochondria could significantly contribute to whole-heart ATP production.

\section{Is there evidence of benefit in patients?}

The positive results in animals during reperfusion after ischemia led to a pilot study in pediatric patients (2). Cardiac mitochondrial transplantation was performed in five children suffering from myocardial ischemia secondary to cardiac surgery and requiring treatment with extracorporeal membrane oxygenation (ECMO) for more than 24 hours. Mitochondria were isolated from rectus abdominis muscle and injected into the ischemic myocardium. All five patients showed qualitative improvement in left ventricular function within days, without shortterm complications. One patient died, whereas the others experienced a recovery of cardiac function enabling ECMO decannulation a few days after reperfusion (2). Because the study was so small, it is unclear whether the published 20\% mortality rate with mitochondrial transplantation is an improvement over the $38 \%-40 \%$ overall mortality rate for pediatric patients on ECMO in large hospitals (18). Additional findings of the group, reported in the media, include three deaths in a total of 11 treated patients $(27 \%)$ and referred to an unusually high $65 \%$ mortality rate in untreated patients for comparison (3). At best, these non-peer-reviewed, anecdotal reports should be viewed with caution.

Importantly, the procedure performed in patients was very different from protocols used in the animal studies. The pediatric patients required ECMO because of ischemic injury that occurred secondary to cardiac surgery. These children received mitochondrial transplantation from two to 15 days after the ischemic injury (2), which is fundamentally different from delivering mitochondria one minute before or after cardiac reperfusion. The purported mechanisms of how mitochondrial transplantation could have improved cardiac function in animal models of ischemia-reperfusion are definitely not applicable to this situation, in which ischemic injury is already complete, and therefore the rationale for undertaking the study is unclear. Furthermore, this study is affected by several other major design flaws, including (a) the absence of a control group; (b) the subjective quantification of ventricular dysfunction; and (c) the lack of uniform criteria to assess cardiac ischemia (2).

\section{Conclusions}

Several critical points make us question whether the procedure of mitochondrial transplantation was sufficiently vetted prior to its application in humans. First, it has not been explained how mitochondria could survive the high $\mathrm{Ca}^{2+}$ extracellular milieu and remain viable, and there is no evidence that viability is maintained. Second, in animal studies, only a miniscule number of mitochondria are found to be colocalized with cardiac cells, let alone internalized far too few to impact tissue ATP production. Third, even if the extracellular mitochondria remained viable and were producing ATP, this ATP would not be available to boost cardiac contractile function, which is inconsistent with rapid improvements in the hemodynamic parameters described in the animal studies (4-6).

Therefore, we would like to express our concern regarding the recent application of this technique to pediatric patients and the increasing media coverage of mitochondrial transplantation. The findings should be replicated in blinded studies in animals first, preferably by independent groups. Notably, other "postconditioning" cardioprotective protocols, despite convincing initial preclinical evidence, failed when subjected to rigorous testing following blinded preclinical study designs $(19,20)$, and, to our knowledge, all have failed in randomized human clinical trials $(21,22)$. The effects of mitochondrial transplantation on the recovery of cardiac function after controlled ischemia-reperfusion are potentially important, and putative mechanisms should be explored. Nevertheless, the extension of these results to a very different protocol in humans, the qualitative and statistically underpowered reports of benefits, the lack of a dose-dependent effect, and the unanswered questions about underlying mechanisms should be viewed skeptically before the miraculous claims trigger a wave of popup clinics for mitochondrial transplantation.

\section{Acknowledgments}

$\mathrm{CM}$ is supported by the Deutsche Forschungsgemeinschaft (DFG) (Ma 2528/7-1, SFB-894, TRR-219) and the Bundesministerium für Bildung und Forschung (BMBF) (01EO1504). BOR is supported by NIH grants (R01HL108917 and R01HL137259). We thank Vasco Sequeira (Comprehensive Heart Failure Center, University Clinic Würzburg, Würzburg, Germany) for technical assistance with the figure.

Address correspondence to: Christoph Maack, Deutsches Zentrum für Herzinsuffizienz, Universitätsklinikum Würzburg, Am Schwarzenberg 15, Haus A15, 97078 Würzburg, Germany. Email: Maack_C@ukw.de. Or to: Brian O'Rourke, Vice Chair of Basic and Translational Research, Department of Medicine, 720 Rutland Avenue, 1060 Ross Building, Baltimore, Maryland 21205-2195; USA.Email: bor@jhmi.edu.

1. Shin B, Cowan DB, Emani SM, Del Nido PJ, McCully JD. Mitochondrial transplantation in myocardial ischemia and reperfusion injury. Adv Exp Med Biol. 2017;982:595-619.

2. Emani SM, Piekarski BL, Harrild D, Del Nido PJ, McCully JD. Autologous mitochondrial transplantation for dysfunction after ischemia-reperfusion injury. J Thorac Cardiovasc Surg. 2017;154(1):286-289.

3. Kolata G. Dying organs restored to life in novel experiments. The New York Times. https:// www.nytimes.com/2018/07/10/health/mitochondria-transplant-heart-attack.html. Published July 10, 2018. Accessed October 23, 2018

4. McCully JD, Cowan DB, Pacak CA, Toumpoulis IK, Dayalan H, Levitsky S. Injection of isolated mitochondria during early reperfusion for cardioprotection. Am J Physiol Heart Circ Physiol. 2009;296(1):H94-H105.

5. Masuzawa A, et al. Transplantation of autologously derived mitochondria protects the heart from ischemia-reperfusion injury. Am J Physiol Heart Circ Physiol. 2013;304(7):H966-H982.

6. Cowan DB, et al. Intracoronary Delivery of Mitochondria to the Ischemic Heart for Cardioprotection. PLoS ONE. 2016;11(8):e0160889.

7. Kaza AK, et al. Myocardial rescue with autologous mitochondrial transplantation in a porcine model of ischemia/reperfusion. J Thorac Cardiovasc Surg. 2017;153(4):934-943.

8. Gunter TE, Pfeiffer DR. Mechanisms by which mitochondria transport calcium. Am J Physiol. 1990;258(5 Pt 1):C755-C786.

9. Preble JM, Pacak CA, Kondo H, MacKay AA, Cowan DB, McCully JD. Rapid isolation and purification of mitochondria for transplantation by tissue dissociation and differential filtration. J Vis Exp. 2014;(91):e51682.

10. Terrovitis J, et al. Magnetic resonance imaging overestimates ferumoxide-labeled stem cell survival after transplantation in the heart. Circu- 
lation. 2008;117(12):1555-1562.

11. Bertero E, Maack C. Calcium signaling and reactive oxygen species in mitochondria. Circ Res. 2018;122(10):1460-1478.

12. Pacak CA, et al. Actin-dependent mitochondrial internalization in cardiomyocytes: evidence for rescue of mitochondrial function. Biol Open. 2015;4(5):622-626.

13. Cowan DB, Yao R, Thedsanamoorthy JK, Zurakowski D, Del Nido PJ, McCully JD. Transit and integration of extracellular mitochondria in human heart cells. Sci Rep. 2017;7(1):17450.

14. Clark MA, Shay JW. Mitochondrial transformation of mammalian cells. Nature. 1982;295(5850):605-607.

15. Kesner EE, Saada-Reich A, Lorberboum-Galski $\mathrm{H}$. Characteristics of mitochondrial transforma- tion into human cells. Sci Rep. 2016;6:26057.

16. Kitani T, Kami D, Matoba S, Gojo S. Internalization of isolated functional mitochondria: involvement of macropinocytosis. J Cell Mol Med.2014;18(8):1694-1703.

17. Katrangi E, et al. Xenogenic transfer of isolated murine mitochondria into human rhoO cells can improve respiratory function. Rejuvenation Res. 2007;10(4):561-570.

18. Nasr VG, Faraoni D, DiNardo JA, Thiagarajan RR. Association of hospital structure and complications with mortality after pediatric extracorporeal membrane oxygenation. Pediatr Crit Care Med. 2016;17(7):684-691.

19. Jones SP, et al. The NHLBI-sponsored Consortium for preclinicAl assESsment of cARdioprotective therapies (CAESAR): a new paradigm for rigorous, accurate, and reproducible evaluation of putative infarct-sparing interventions in mice, rabbits, and pigs. Circ Res. 2015;116(4):572-586.

20. Lefer DJ, et al. Sodium nitrite fails to limit myocardial infarct size: results from the CAESAR Cardioprotection Consortium. FASEB J. 2014;28(1_Suppl):LB645.

21. Engstrøm T, et al. Effect of ischemic postconditioning during primary percutaneous coronary intervention for patients with ST-segment elevation myocardial infarction: a randomized clinical trial. JAMA Cardiol. 2017;2(5):490-497.

22. Hausenloy DJ, et al. Remote ischemic preconditioning and outcomes of cardiac surgery. $N$ EnglJ Med. 2015;373(15):1408-1417. 